\title{
Les bases phyto-écologiques de la lutte anticulicidienne
}

\section{Cartographie des biotopes larvaires}

\section{Ses applications opérationnelles dans le "Midi" méditerranéen}

\author{
par Jean-A. RIOUX, Henri CROSET, Jean-J. CORRE, \\ Pierre SIMONEAU et Georges GRAS
}

Caractériser un biotope, c'est-à-dire exprimer de manière claire et concise sa physionomie, ses limites et sa dynamique, représente l'une des difficultés majeures de l'Ecologie, singulièrement dans ses applications à l'étude des vecteurs, des hôtes intermédiaires et des réservoirs de virus.

Et cependant, malgré les obstacles, une telle expression est souhaitable, voire indispensable, en particulier lors d'enquêtes épidémiologiques, si l'on veut donner une interprétation correcte des facteurs limitants et des cycles évolutifs et, plus encore si l'on doit planifier, dans le temps et l'espace, les opérations de lutte proprement dite.

Dans cet esprit, essentiellement pratique, nous nous proposons d'exposer les motifs qui nous ont conduits à adopter la couverture végétale comme indicateur écologique des gîtes larvaires de Culicides. L'application effective de cette méthode, réalisée dans le sud de la France (1) à partir de 1963 pour l'ensemble des départements côtiers du Languedoc-Roussillon $\left(27.800 \mathrm{~km}^{2}\right)$, nous a permis de lever une carte écologique au $1 / 5.000^{\circ}$ dont l'intérêt n'a cessé de se confirmer au cours des campagnes successives (2).

(1) Grâce à l'aide de l'E.I.D.

(2) Cette méthode a d'ailleurs fait ses preuves en d'autres domaines tels l'épidémiologie de la peste selvatique (Y.-J. Golvan et J.-A. Rioux, 1963) ou le dépistage des gîtes de ponte du Criquet pèlerin, Schistocerca gregaria (G. Popov, W. Zeller et J. Cochème, 1964). 


\section{I. - Rappel des principaux biotopes culicidiens du sud de la France}

Avant d'aborder les problèmes proprement cartographiques, il convient de rappeler brièvement les caractères des principaux gîtes larvaires de Culicides observés dans le « Midi » de la France.

Schématiquement, les deux grands types de gîtes, urbains et ruraux, peuvent être distingués.

A. - Les gîtes urbains, anthropogènes, sont essentiellement colonisés par Culex pipiens s.l. Ils comprennent des biotopes hypogés, riches en matières organiques (caves inondées, fosses) où pullule la forme autogène et homodyname, et des biotopes épigés (bassins, fossés, égouts à ciel ouvert...), où se développe la forme anautogène et hétérodyname.

B. - Les gîtes ruraux sont le plus souvent naturels. Leur dynamique permet de les diviser en deux grands types écologiques, permanents et temporaires :

$1^{\circ}$ Les biotopes permanents (3) sont généralement dulçaquicoles parfois légèrement saumâtres. Dans le «Midi » méditerranéen, les plus riches en matières organiques sont colonisés par la forme anautogène du Culex pipiens, les autres par Culex modestus, Anopheles maculipennis, Anopheles labranchiae atroparvus et Anopheles hyrcanus. Enfin, Culiseta subochrea et Anopheles algeriensis habitent les gîtes légèrement saumâtres.

$2^{\circ}$ Les biotopes temporaires sont colonisés par une série d'espèces dont certaines sont dotées de caractères adaptatifs frappants. En particulier, leurs œufs, déposés sur le sol humide, peuvent demeurer quiescents pendant de longues périodes. Ils n'éclosent, lors des mises en eau, que sous certaines conditions physico-chimiques (température, $p \mathrm{H}$, $r \mathrm{H}$...). Le développement larvaire en est alors très souvent explosif (cycle inférieur à 6 jours pour Aedes caspius).

Les biotopes temporaires dulçaquicoles sont faiblement représentés sur les basses terres du littoral méditerranéen. Citons parmi ceux-ci, les prairies inondables, gîtes à Aedes vexans, les creux de rochers, en bordure de cours d'eau, gîtes à Aedes vittatus, les creux d'arbres, gîtes à Anopheles plumbeus, Aedes geniculatus, Aedes berlandi, Aedes pulchritarsis et Orthopodomyia pulchripalpis.

Les biotopes temporaires halins, objet principal de notre étude, occupent, par contre, de très grandes surfaces. Pour l'ensemble de la région côtière, on peut en distinguer deux types, les creux de rochers sub-littoraux, gîtes à Aedes mariae et les marais saumâtres des basses plaines alluviales, gîte à Aedes caspius et Aedes detritus (fig. 3, 4 et 5).

(3) La notion de gîte permanent est évidemment liée au comportement de l'espèce. Ainsi, la rizière peut être un gîte permanent au cours de phase de pleine activité (Anopheles maculipennis s.1. et Culex modestus) ou un gîte temporaire au moment de la mise en eau ou, dans le cas d'abandon, au moment des pluies (Aedes vexans et Aedes caspius). 
Du point de vue écologique, Aedes detritus et Aedes mariae se comportent comme des espèces halobies, c'est-à-dire inféodées aux gîtes les plus chargés en chlorures (10 à $150 \mathrm{~g}$ de $\mathrm{NaCl}$ par litre). Les œufs d'Aedes detritus éclosent de septembre à avril. Aedes caspius, plus ubiquiste, peut être considéré comme une espèce simplement halophile, susceptible de coloniser une gamme beaucoup plus étendue de biotopes saumâtres. Elle se développe en saison chaude, de mars à octobre, avec deux pointes de pullulation, vernale et automnale.

\section{II. - La végétation, révélateur écologique}

Au demeurant, s'il est assez facile de définir, de délimiter, et, en dernière analyse, de cartographier les milieux aquatiques permanents, il n'en est pas de même des temporaires et plus particulièrement des milieux lagunaires, instables par essence. Ici, les données physico-chimiques et surtout topographiques perdent une grande partie de leur intérêt en raison des importantes fluctuations saisonnières, voire journalières, qu'elles présentent. Par contre, la méthode phyto-écologique, basée sur l'étude de la végétation phanérogamique, apporte une série de critères de valeur dont certains d'une grande portée pratique.

De fait, la végétation fait partie du milieu qu'elle modifie souvent de façon non négligeable (pédogenèse, micro-climat). A leur tour, les plantes sont conditionnées par le milieu (texture, structure et hydromorphie des sols, rythme et durée de submersion) qui trie les espèces les mieux adaptées et façonne en quelque sorte le paysage végétal. En définitive, la végétation, véritable intégrateur, représente l'expression la mieux équilibrée et la plus stable des divers facteurs écologiques. Elle est, selon l'expression de Charles Flahaut, « le miroir du milieu ». Cartographier la végétation revient donc à matérialiser la résultante des divers éléments du milieu, tant physico-chimiques que biotiques.

\section{III. - Végétation et biotopes culicidiens}

\section{A. - Généralités.}

Dans certains cas, malheureusement trop rares au gré des écologistes, la végétation représente, pour la larve de Culicide, le substrat direct. C'est le cas, en particulier, des espèces du genre Mansonia, liées à la présence de certains végétaux aquatiques (Typha, Phragmites). De même, pour la faune dendro-limnique (Orthopodomyia, Anopheles plumbeus), l'Arbre constitue le biotope lui-même.

Mais la végétation intervient aussi sur l'imago: ainsi, dans les hautes montagnes des Pyrénées-Orientales, Aedes cataphylla, Aedes pullatus, Aedes excrucians et Aedes punctor, qui pullulent à l'étage sub-alpin, ne semblent pas déborder sur l'étage alpin, bien qu'on y observe des milieux aquatiques très semblables. La différence essentielle tient, semble-t-il, à la répartition du Pin à crochet (Pinus uncinata) caractéristique de l'étage 
sub-alpin. Sous le couvert de la forêt (sous-bois à Rhododendron, Genista et Juniperus) s'établit un micro-climat propice à la survie des adultes alors que l'étage alpin, dépourvu de strate arborescente, constitue un milieu hautement xérique, très défavorable aux imagos (vent violent, forte insolation, sécheresse atmosphérique).

Cependant, lorsque la corrélation «biotope-végétation 》n'est pas aussi évidente, par exemple dans les marais lagunaires, une étude plus poussée est nécessaire.

Ainsi, sur le littoral méditerranéen, la recherche des œufs d'Aedes dans le sol par la méthode de W. R. Horsfall a permis de préciser sans ambiguïté les liaisons entre les gîtes larvaires et les végẹtaux phanérogamiques. Rappelons succinctement la technique suivie et les résultats obtenus :

\section{B. - Extraction des oufs d'Aedes dans les sols salés.}

$1^{\circ}$ Technique : les opérations se déroulent en 5 phases:

\section{Prélèvement de l'échantillon :}

Le matériel de prélèvement est relativement simple; dans le cas de gîtes exondés, on utilise une truelle à joints de 10 à $15 \mathrm{~cm}$ de long, à bords tranchants ainsi qu'une planchette carrée de $15 \mathrm{~cm}$ de côté. Celle-ci, placée à plat sur le sol permet de découper un parallélépipède de terre de $2,5 \mathrm{~cm}$ d'arête environ. L'échantillon, détaché du sol, est directement placé dans un sac de papier ou de nylon. Dans les cas de gîtes inondés, il est nécessaire d'adjoindre un carré tranchant de $15 \mathrm{~cm}$ de côté ainsi qu'une pelle plate. Le carré, sorte de moule, est enfoncé à plat dans le sol. La pelle est alors glissée au ras des bords de l'échantillon prélevé avec précaution. Dès leur prélèvement, les échantillons doivent être conservés en chambre froide jusqu'à leur traitement au laboratoire.

\section{Tamisage :}

L'appareil utilisé pour cette opération, comporte deux éléments essentiels (fig. 1):

a) Un ensemble de trois cylindres emboîtés, formés de grillage dont les mailles décroissent de l'intérieur $\left(15,6 \mathrm{~mm}^{2}\right)$ à l'extérieur $\left(3,9 \mathrm{~mm}^{2}\right)$. Ces cylindres, solidaires, tournent horizontalement dans un bain d'eau douce, entraînés par un moteur à la vitesse de 50 tours/minute. L'ensemble est fermé à chaque extrémité par un couvercle métallique plein, solidaire de l'axe de rotation.

L'échantillon, placé à l'intérieur du cyclindre, est alors soumis à trois séries de 25 rotations dans chaque sens. L'opération terminée, la cuve est vidée sur un système de 4 tamis par un tube caoutchouc de fort calibre.

b) Le jeu de tamis comprend 4 éléments superposés dont la taille des mailles est de $0,39 \mathrm{~mm}^{2}, 0,18 \mathrm{~mm}^{2}$ et $0,062 \mathrm{~mm}^{2}$. Dans le tamis supérieur, sont retenus les sables grossiers; les tamis moyens arrêtent les sables fins; seul, le tamis inférieur retient les œufs. Les argiles et le limon sont entraînés avec l'eau de vidange. Un jet puissant permet un rinçage soigneux des parois à chaque phase de l'opération. 


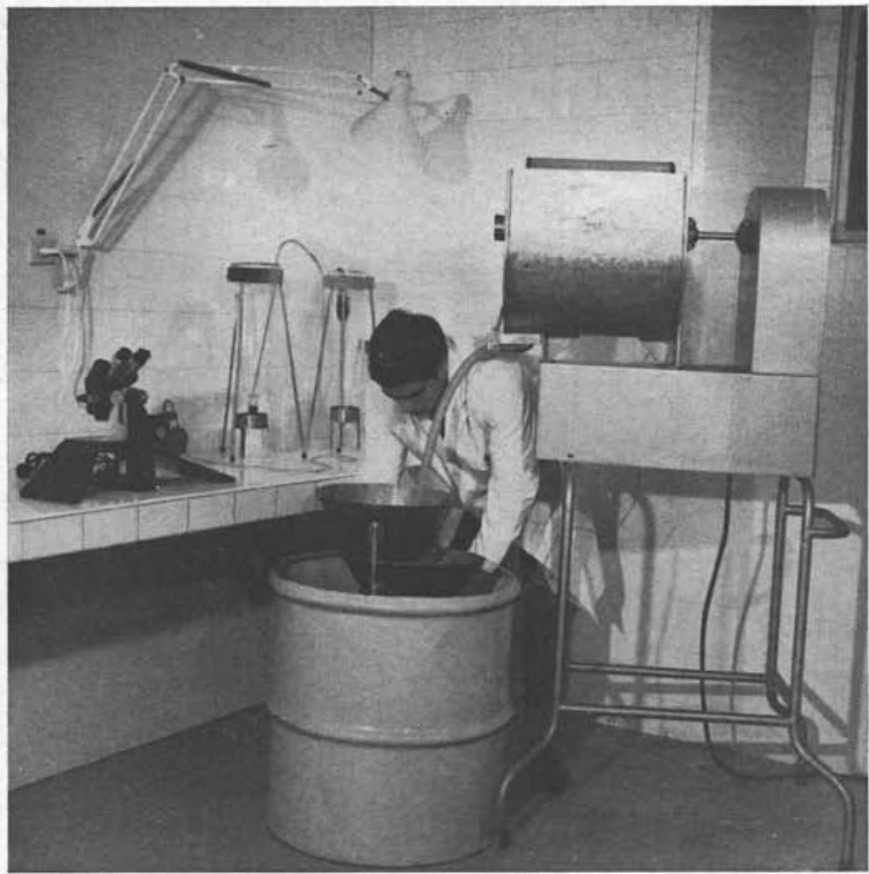

FIg. 1. - Appareil de Horsfall pour l'extraction des aufs du sol

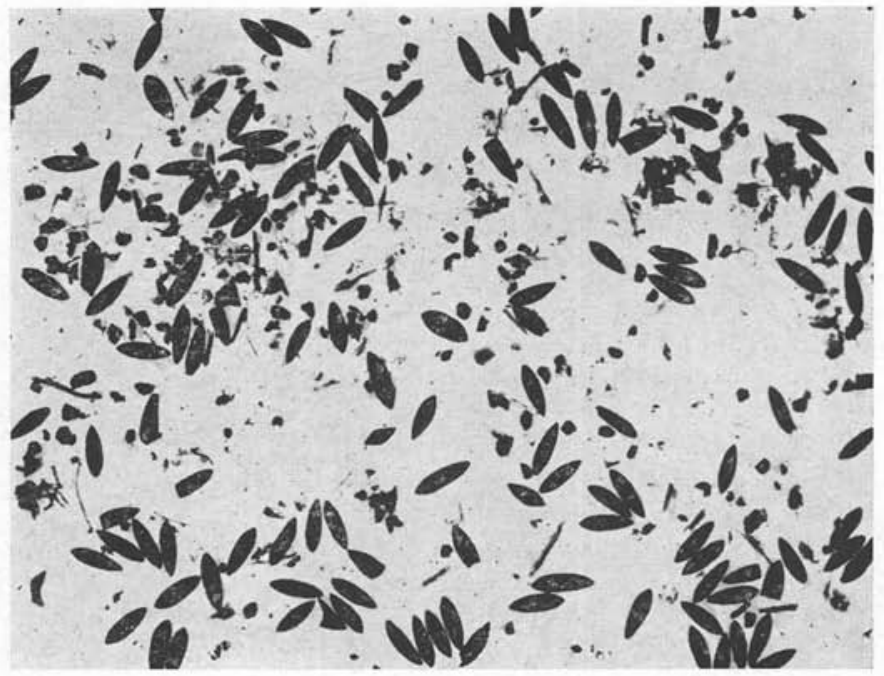

Fig. 2. - CEufs d'Aedes detritus extraits d'un échantillon de sol par la technique de Horsfall 


\section{Flottation et Sédimentation :}

Le filtrat contenant les œufs est remis en suspension dans un entonnoir à décantation rempli d'une solution saturée de $\mathrm{NaCl}$. Un aérateur à bulles, alimenté par une pompe d'aquarium, provoque la flottation des œufs (4), alors que les éléments plus denses précipitent ou restent au fond. En fin d'opération, l'entonnoir est ouvert à sa partie inférieure et les éléments grossiers sont éliminés. L'opération est renouvelée une fois ou deux.

\section{Tri optique :}

Le liquide restant est filtré sur papier Joseph, puis trié (fig. 2) sous la loupe binoculaire (5).

\section{Détermination :}

L'identification des espèces est possible par l'étude de la structure de l'endochorion. L'embryon est au préalable éliminé avec une aiguille montée et l'endochorion éclairci par la méthode de G. B. Graig (6).

La forme, la taille et la structure des aréoles, caractéristiques de l'espèce, sont alors visibles directement au microscope en lumière transmise ou réfléchie.

\section{$2^{\circ}$ RÉsultats.}

Les indications de la méthode sont multiples. Dans le cadre particulier de notre étude, elle a permis de montrer l'existence d'une étroite corrélation entre végétation phanérogamique et gîtes de ponte. Ainsi, les marais à Juncus maritimus ou Salicornia fruticosa abritent un nombre considérable d'œufs, tandis que les phytocénoses voisines n'en hébergent souvent que quelques unités.

Le transect suivant, réalisé en Camargue, au lieu-dit Mas Astouin, le 20 avril 1965, illustre le fait :

Prélèvement $n^{\circ} 1$ : Bordure supérieure du gîte.

Végétation: Agropyrum acutum.

Densité d'œufs : $0 / d m^{2}$.

Prélèvement $n^{\circ} 2$ : Pente à $0,20 \mathrm{~m}$ en contrebas du précédent.

Végétation : Juncus maritimus.

Densité d'œufs : $65 / \mathrm{dm}^{2}$.

Prélèvement $n^{\circ} 3$ : Limite inférieure de la bordure à Joncs.

Végétation: Juncus maritimus clairsemé + Suada maritima.

Densité d'œufs : $4 / d m^{2}$.

(4) Les microbulles d'air retenues dans les aréoles d'air allègent l'œuf et l'entraînent en surface.

(5) Ainsi que l'indique W.R. Horsfall, 85 à $90 \%$ des œufs préalablement mélangés à un sol vierge sont retrouvés en fin d'opération.

(6) Dans cette méthode, la décoloration du chorion est obtenue par le chlore naissant (dégagé par la réaction de $\mathrm{ClH}$ sur $\mathrm{ClO}_{3} \mathrm{~K}$ ). 
Prélèvement $n^{\circ} 4$ : Bas-fond à $0,20 \mathrm{~m}$ en contrebas du précédent.

Végétation : croûte de Cyanophycées.

Densité d'œufs : $0 / \mathrm{dm}^{2}$.

Les prélèvements effectués au centre même du gîte, sont tous négatifs. Ainsi dans ce biotope, de plus de 100 mètres de large, les œufs d'Aedes sont strictement localisés à la frange de Juncus maritimus, large seulement de 0,30 à $0,50 \mathrm{~m}$.

\section{IV. - Cartographie phyto-écologique}

\section{$1^{\circ}$ Choix des indicateurs.}

Le nombre même des méthodes proposées pour l'étude et l'expression cartographique de la végétation montre, à l'évidence, les difficultés du problème.

Dans la méthode phyto-sociologique (J. Braun-Blanquet), la plus utilisée en Europe occidentale, la source d'information est le « relevé » obtenu par l'échantillonnage quantitatif d'une surface homogène et d'étendue suffisante pour être représentative de la phytocénose. Le relevé, comportant souvent plusieurs dizaines d'espèces, est complété par une série de renseignements sur le milieu (situation géographique, géomorphologie, pédologie, géologie...). La comparaison des relevés provenant de phytocénoses semblables permet par le jeu de tableaux, de dégager l'Association végétale correspondante.

La méthode des groupes écologiques statistiques (M. Gounot) est actuellement très employée (7). Elle se révèle d'une plus grande précision car le groupe écologique représente un ensemble d'espèces « ayant un comportement écologique semblable » et des affinités sociologiques statistiquement définies.

La méthode des «transects», basée sur l'échantillonnage linéaire de la végétation, permet de suivre les modifications du tapis végétal parallèlement aux variations des facteurs écologiques (microtopographie, humidité superficielle, texture, fluctuation de la nappe, etc.). Très utile pour interpréter les rapports plante-substrat, elle est, par essence, sans intérêt direct pour l'établissement de la carte proprement dite.

Pour plusieurs raisons, dont les plus importantes d'ordre pratique, et tout en tenant compte des données précédentes, nous avons adopté une méthode éclectique, essentiellement basée sur la physionomie végétale. Dans notre carte phyto-écologique, chaque groupement est défini par une ou plusieurs plantes dominantes, visibles à l'observateur dès le premier abord. Si nécessaire, l'indication de quelques espèces compagnes, permet de préciser les nuances écologiques supplémentaires telles l'hydromorphie, l'halomorphie et la texture du sol.

(7) La conception de $\mathrm{M}$. Gounot est la synthèse de la notion de groupe écologique au sens de Duvigneaud et des études auto-écologiques d'Ellenberg. 


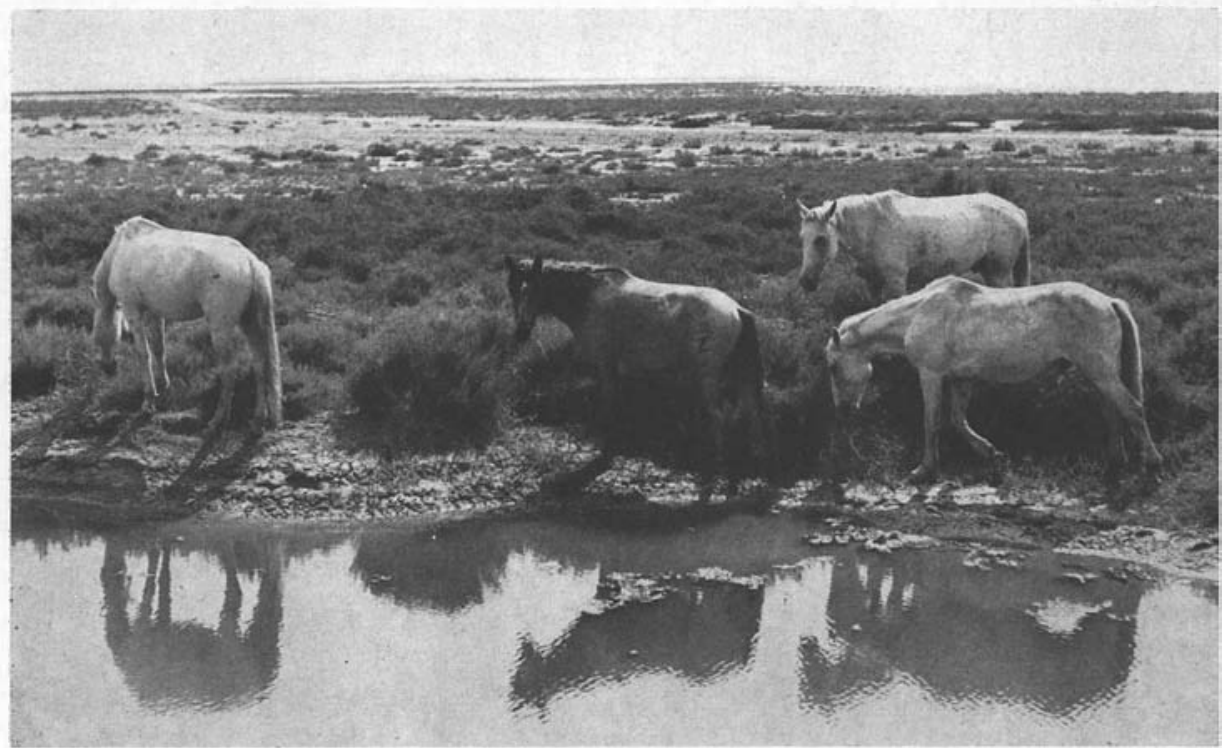

FIG. 3. - «Sansouires » à Salicornia fruticosa, Salicornia radicans et Art'irocnemum glaucum. Gîte à Aedes detritus et Aedes caspius (cliché Georges, Arles)

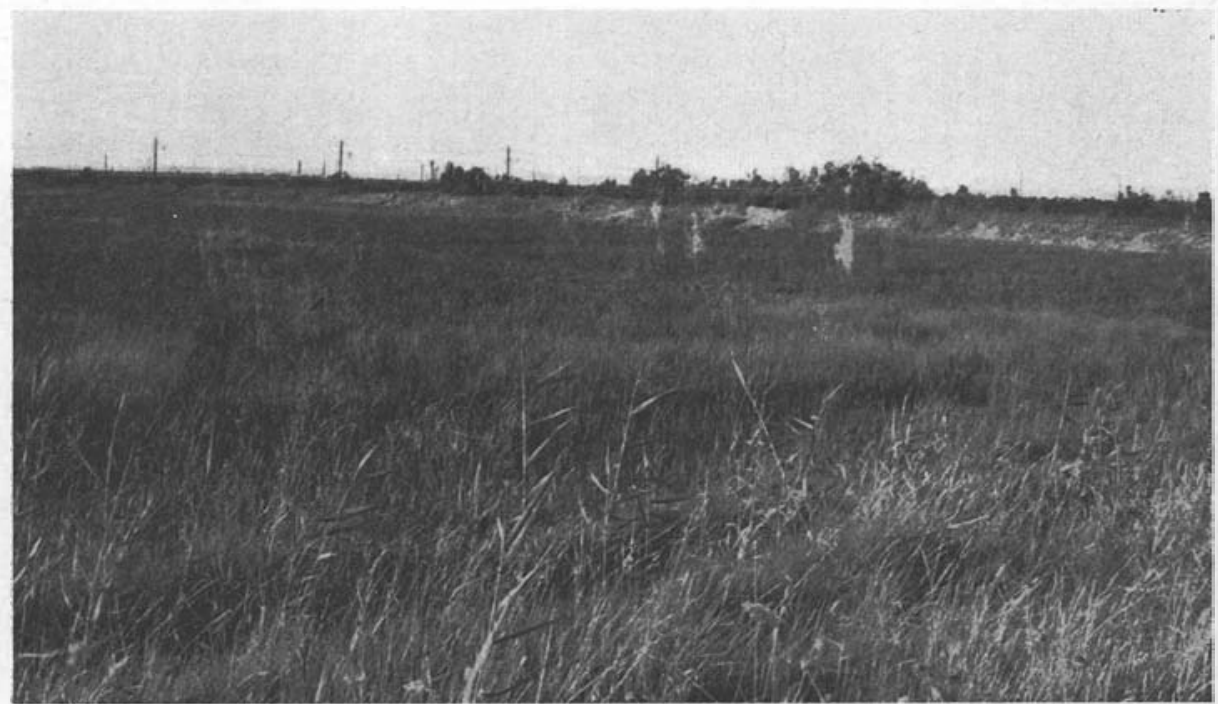

FIG. 4. - Phragmitaie claire et Jonçaie halophile, gite verno-automnal à Aedes caspius 
Ce faisant, nous avons pu individualiser une trentaine de groupements classés eux-mêmes en quatre grandes unités :

- les «Sansouires », essentiellement halomorphes,

— les « Roselières », hydromorphes,

— les «Jonçaies»,

- les «Prés salés » à hydromorphie et halomorphie faibles.

Dans chacune de ces unités, les groupements ont été classés par ordre décroissant d'hydromorphie.

A. Dans l'unité «SANSourRE », on peut distinguer (fig. 3):

- Groupement à Rupia maritima des étangs saumâtres à submersion quasi permanente.

- Groupement à Salicornia herbacea $(1 \mathrm{~A})$ *.

- Groupement à Salicornia radicans (61).

- Groupement à Arthrocnemum glaucum (forme érigée).

- Groupement à Salicornia fruticosa variante à Juncus subulatus (3).

- Groupement à Salicornia fruticosa variante à Juncus maritimus (1 C).

- Groupement à Salicornia fruticosa s. st. (1).

- Groupement à Salicornia fruticosa et Arthrocnemum glaucum (4).

- Groupement à Salicornia fruticosa et Aeluropus littoralis.

- Groupement à Salicornia fruticosa variante à Plantago coronopus (2).

- Groupement à Arthrocnemum glaucum forme prostrée (5).

- Groupement à Inula crithmoides et Juncus maritimus (9).

- Groupement à Suaeda fruticosa et Obione portulacoides (8).

- Groupement à Suaeda fruticosa et nitrophiles (8 A).

B. Dans l'unité « Roselière », composée d'espèces sensiblement moins halophiles voire dulçaquicoles, on peut distinguer (fig. 4) :

- Groupement à Phragmites communis et Typha angustifolia ou Typha latifolia des eaux douces, profondes et stagnantes.

- Groupement à Phragmites communis et Scirpus lacustris vicariant du précédent dans les eaux courantes ou tout au moins mieux aérées (22).

- Groupement à Phragmites communis et Scirpus maritimus des eaux stagnantes, légèrement salées (20).

- Groupement à Phragmites communis et Juncus maritimus.

- Groupement à Phragmites communis et Aster tripolium (19).

- Groupement à Phragmites communis et Bonjeania recta des eaux douces et renouvelées (cours d'eau, ripisylve) (23 A).

- Groupement à Scirpus maritimus et Aeluropus littoralis vicariant du précédent en zone faiblement halophile.

* Les numéros des groupements renvoient à la carte générale de la végétation au 1/5.000 du littoral Languedoc-Roussillon. 
C. Dans l'unité «JONÇAIE », écologiquement moins homogène, on peut distinguer les groupements suivants (fig. 5).

- Groupement à Juncus maritimus pur (J. M.).

- Groupement à Juncus subulatus et Aeluropus littoralis (18).

- Groupement à Juncus maritimus et Triglochin maritimum (27).

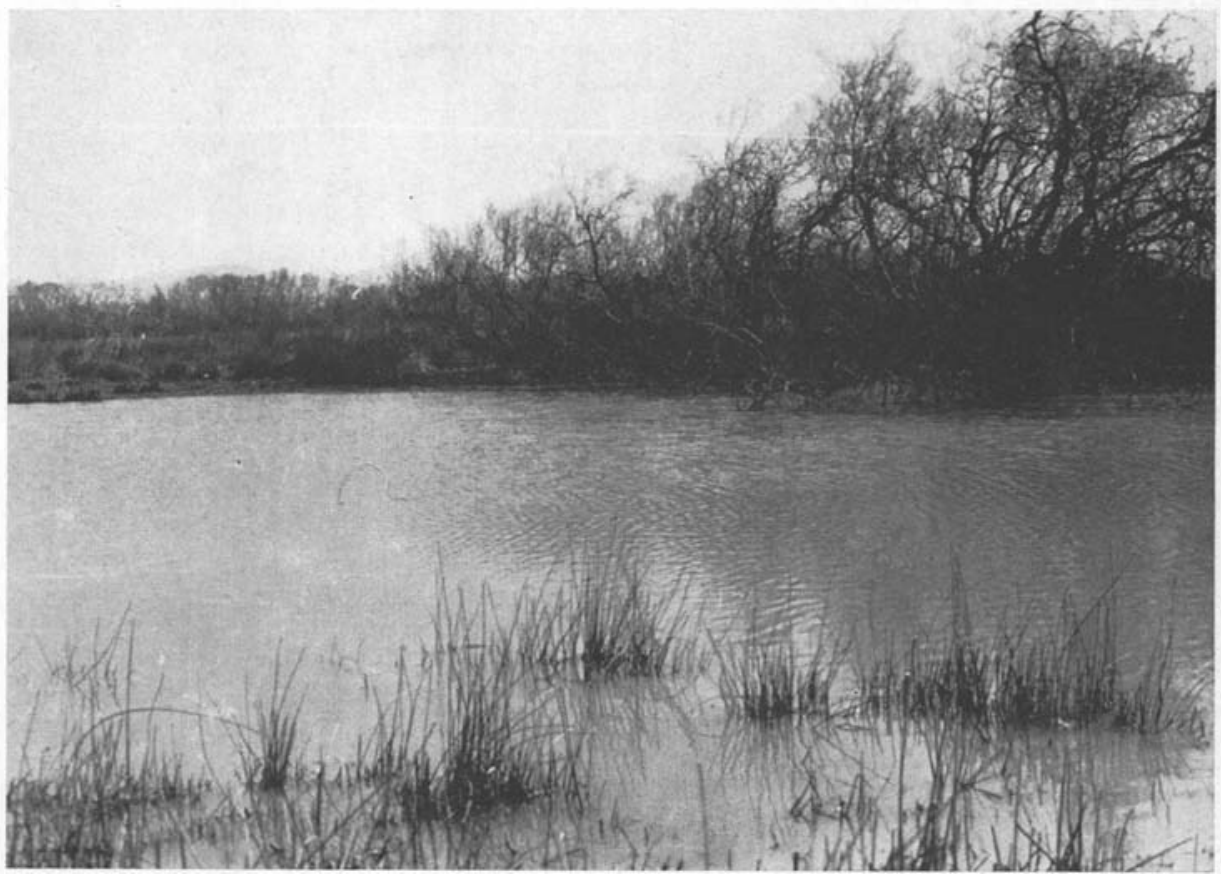

FIG. 5. - Jonçaie inondée, gîte verno-automnal à Aedes caspius

- Groupement à Juncus maritimus et Schoenus nigricans vicariant du précédent s'ur sol sableux (29).

- Groupement à Juncus maritimus et Aeluropus littoralis (17).

D. L'unité «Pré SALÉ 》 fait en général suite à la «Jonçaie ». On peut y distinguer les types de végétation suivants:

- Groupement à Spartina versicolor (26).

- Groupement à Agropyrum acutum et Juncus maritimus (13).

- Groupement à Agropyrum acutum et Schoenus nigricans, vicariant du précédent sur sol sableux (25).

- Groupement à Glyceria festucaeformis et Lotus decumbens, variante à Obione portulacoides (11). 


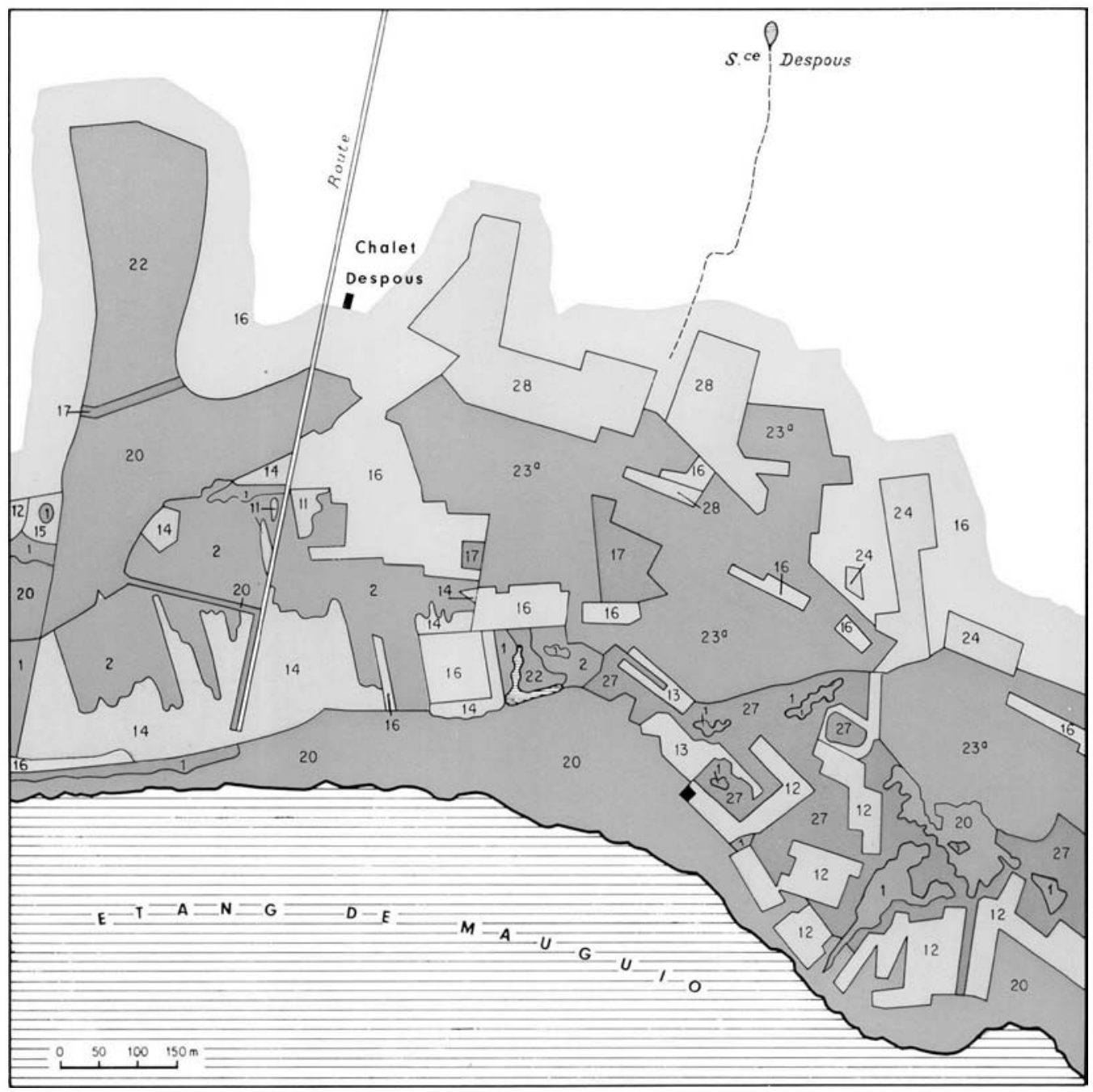

Fig. 7. - Carte phỵto-écologique définitive.

LÉGExbE. - Bleu : roselières. Rouge : “sansouires". Vert foncé : jonçaies. Vert clair : prés salés. Jaune : cultures. 1) Groupement à Salicornia fruticosa. 2) Groupement à Salicornia fruticosa variante à Plantago coronopus. 11) Groupement à Glyceria festucaformis et Lotus decumbens variante à Obione portulacoides. 12) Groupement à Trifolium maritimum et Agropyrum acutum. 13) Groupement à Agropyrum acufum et Juncus maritimus. 14) Groupement à Agropyrrum aculum et Obioue portulacoides. 15) Groupement ì Agrop!yrum acutum et Daucus carota. 16) Cultures ; jachères récentes. 17) Groupement à Aeluropus liftoralis et Juncus marilimus. 20) (iroupement à Phragmites communis et Scirpus maritimus. 22) Groupement à Phragmites communis et Scirpus lacustris. 23 a) Groupement ì Phragmites communis et Bonjeania recta. 24) Groupement ì Tragopogon pratensis et Trifolium pratense. 27) Groupement à Triglochin marilimum et Juncus maritimus. 28) Groupement it Dorycnium gracile et Schanus nigricans. 
- Groupement à Glyceria festucaeformis et Lotus decumbens (10).

- Groupement à Trifolium maritimum et Agropyrum acutum (12).

- Groupement à Tragopogon pratensis et Trifolium pratense (24).

- Groupement à Agropyrum acutum et Obione portulacoides, à la limite des zones exondées (14).

- Groupement à Agropyrum acutum et Daucus carota (15).

- Groupement à Dorycnium gracile et Schoenus nigricans, vicariant du précédent sur sol sableux (28).

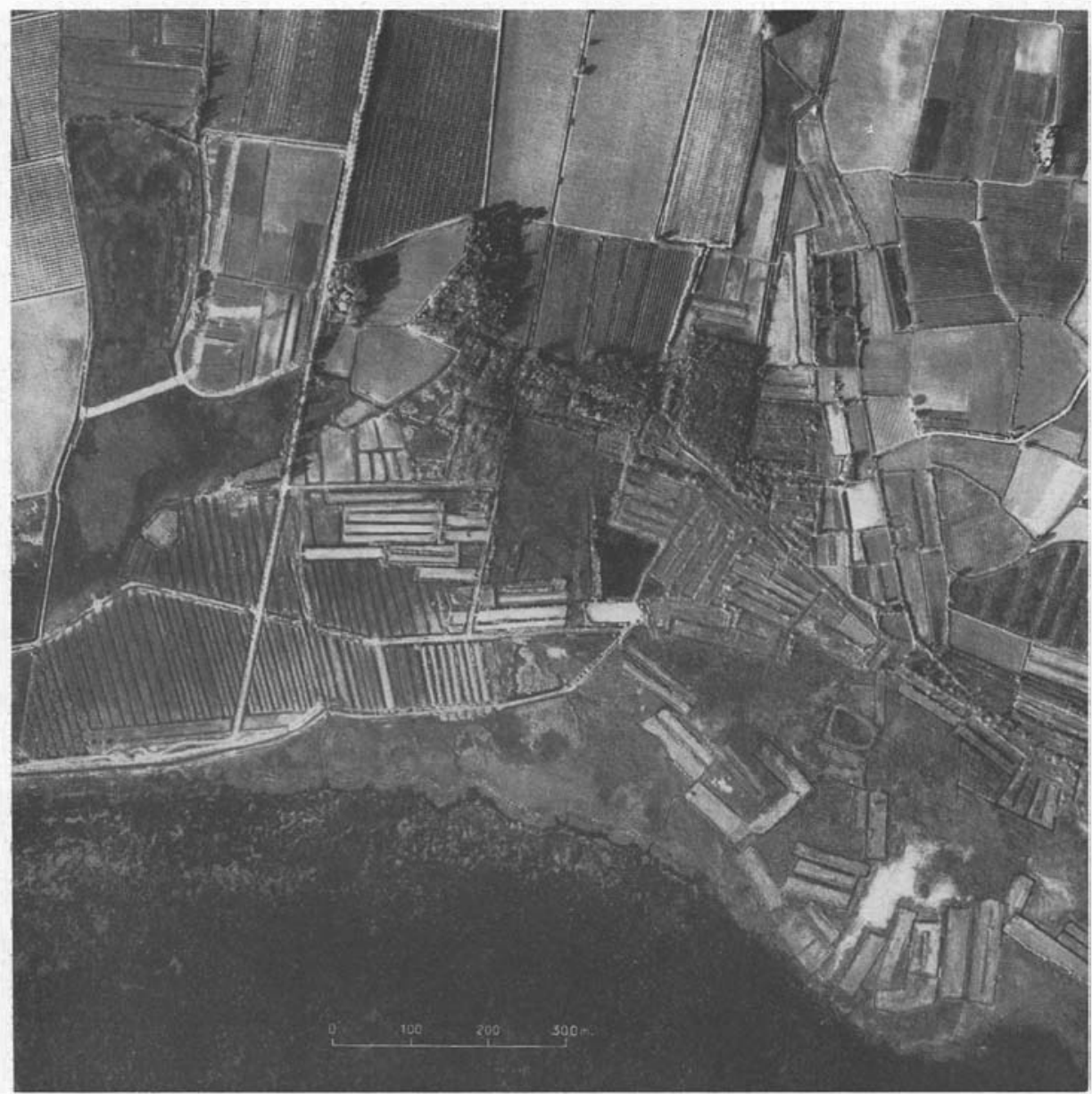

Fig. 6. - Photographie aérienne correspondant à la carte phyto-écologique définitive de la page précédente (zone du Chalet Despous au nord de l'étang de Mauguio, près de Montpellier) 


\section{$2^{\circ}$ Choix de l'échelle. Photo-interprétation. Expression graphique.}

Au stade de l'élaboration, l'un des problèmes majeurs posés au cartographe est sans conteste, celui de l'échelle de représentation. Notre cas ne fait pas exception à la règle. En matière de gîte culicidien, une échelle trop grande risquait d'être peu maniable ; inversement, la précision du tracé devait être suffisante pour permettre le repérage aisé d'un gîte de moins de $50 \mathrm{~m}^{2}$. En définitive, après divers essais, nous avons retenu l'échelle du $1 / 5.000^{\circ}$ qui, outre sa maniabilité, donnait la possibilité non négligeable d'utiliser des symboles complémentaires.

Un deuxième problème, général lui aussi, tient aux difficultés de repérage des limites des groupements. Lors des levées de cartes en terrain plat, le prospecteur se trouve assez souvent au centre d'un groupement dense et élevé (groupement à Phragmites communis par exemple) qui lui cache la plupart des repères topographiques et rend son tracé imprécis. Pour pallier ces difficultés, nous avons utilisé d'emblée les photographies aériennes (fig. 6), soit directement, soit après photo-interprétation. Sur la photographie au $1 / 5.000^{\circ}$ les différents types physionomiques de végétation se distinguent en effet nettement par leurs caractéristiques de structure (lisse, granuleux, strié...) de forme, de relief et de teinte. L'étude des couples stéréoscopiques apporte par ailleurs de nombreux détails supplémentaires.

Pour l'expression graphique proprement dite (fig. 7), en particulier pour ce qui concerne le choix des couleurs, nous nous sommes conformés aux recommandations classiques des botanistes. Ainsi, les teintes rouges ont été réservées aux unités phytoécologiques les plus sèches et les plus salées (Sansouires) ; les teintes bleues, aux unités les plus inondées et les plus douces (Roselières) ; les teintes intermédiaires permettant de nombreuses combinaisons : le violet, par exemple, figure les zones à Scirpus maritimus moins inondées que les zones à Phragmites communis (bleues) et moins halomorphes que les Sansouires à Salicornia fruticosa (rouge).

Sur le plan pratique, la réalisation de la carte phyto-écologique au $1 / 5.000^{\circ} \mathrm{com-}$ porte les opérations suivantes:

- Centralisation des documents (cartes, photographies aériennes, documents hydrologiques, pédologiques, phytosociologiques...).

- Photo-interprétation proprement dite: les photographies intéressant une zone test, sont recouvertes d'un calque transparent et rigide (type Kodatrace). Les contours des unités sont tracés à l'aide d'un stéréoscope à miroir de grossissement 3 à 5 .

- Signification physionomique des unités individualisées sur le document photographique. Cette opération, d'une très grande importance pratique, s'effectue sur le terrain grâce à une série de «transects» réalisés en saison favorable.

- Extension des unités photo-interprétées à l'ensemble de la zone à cartographier.

- Correction sur le terrain des zones litigieuses et contrôle définitif des unités photo-interprétées à l'aide de quelques « transects » judicieusement choisis.

- Report des contours sur la carte définitive.

- Choix des couleurs.

- Tirage éventuel par procédé «Offset». 


\section{$3^{\circ}$ Découpage de la carte phyto-écologique. Notion d'ensemble écologique.}

Les impératifs opérationnels de la lutte anti-larvaire nous ont amenés à découper la carte phyto-écologique en un certain nombre de secteurs autonomes ou « ensembles écologiques » (analogues aux «bassins versants » de nos cours d'eau) dans lesquels les phytocénoses réagissent de la même manière à l'égard des submersions ou des remontées de nappes. Les différences de «fonctionnement», d'un ensemble écologique à l'autre, tiennent essentiellement à l'hétérogénéité géomorphologique de la côte et ses corollaires, les modes de mises en eau et de drainages.

Parmi les principales causes de cette hétérogénéité, citons :

- l'inégale répartition de l'impluvium due à l'étroite localisation des orages (régime torrentiel méditerranéen);

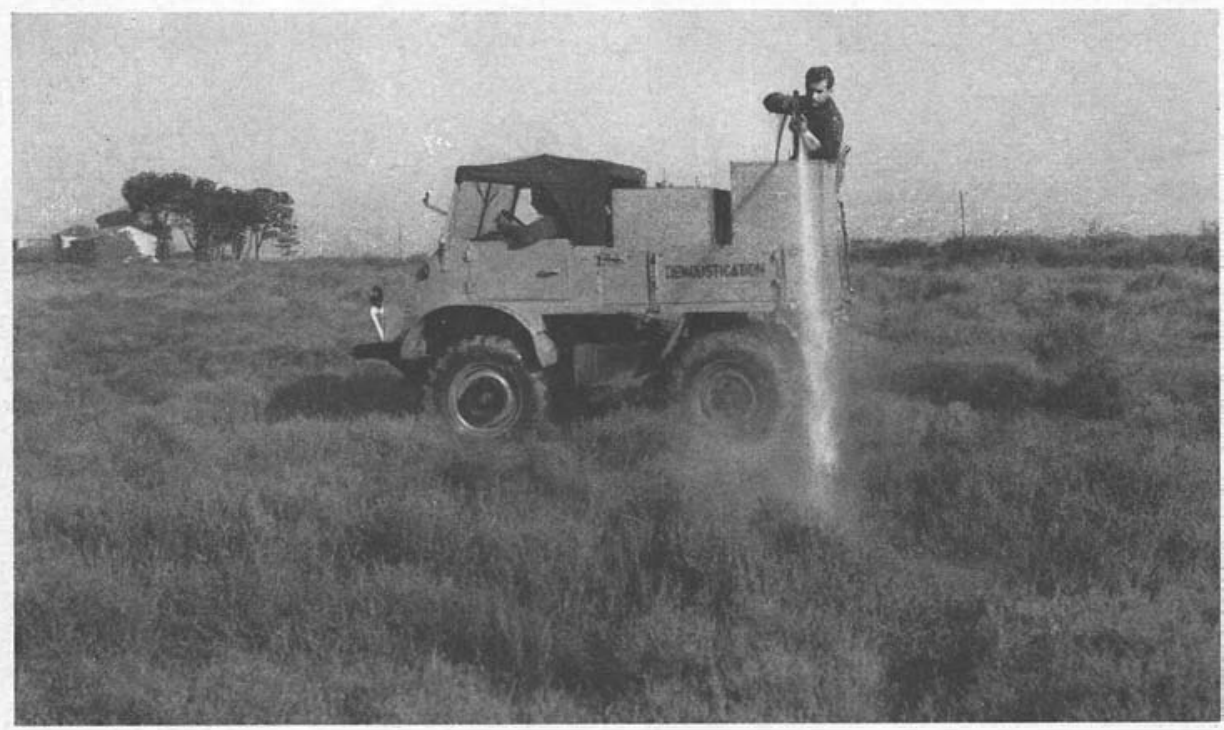

FIG. 8. - Lutte anti-larvaire par épandage rationnel d'insecticide dans unz «sansouire » à Salicornia fruticosa, gîte hivernal à Aedes detritus

- les différences d'orientation de la côte par rapport aux incursions marines (vents du large dominants de secteur Est et Sud);

- la dissociation des crues d'un fleuve à l'autre (en grande partie liée aux deux causes précédentes) ;

— l'hétérogénéité des sols (drainage vertical rapide des sols sableux ; ruissellement ou stagnation sur les sols lourds).

L'individualisation de ces ensemble a permis, sur le plan pratique, de perfectionner les techniques de dépistage des gîtes et de traitement tant anti-larvaire qu'anti-adulte. Il n'est pas jusqu'aux travaux de génie qui n'aient tiré un grand bénéfice de ces notions. 


\section{4- Utilisation de la carte phyto-écologique. Applications pratiques.}

A l'usage, la carte de végétation au $1 / 5.000^{\circ}$ se révèle un précieux instrument de travail aussi bien dans l'élaboration des programmes de lutte que dans la conduite des opérations proprement dites.

Au stade des PRÉvisions, elle permet, par simple planimétrie, de calculer l'étendue des gîtes potentiels et d'estimer ipso facto le volant moyen d'insecticides, de matériels d'épandage et de personnels.

Au stade de la prospection, elle permet de suivre, sans difficulté particulière les fluctuations de la nappe et par là-même, de connaître en quelques heures, la localisation et l'importance des «zones d'éclosion» (8).

Au stade du TRAITEMENT, (fig. 8), elle permet de dégager les indications des diverses méthodes de lutte (doses et formulation de l'insecticide (9), choix de l'appareil de traitement). Ainsi, l'épandage aérien sera utilisé sur les gîtes vastes et ouverts, l'épandage au sol, par véhicules tous terrains, sur les gîtes de petite taille ; les granulés seront préférés aux émulsions pour le traitement des gîtes à végétation verticale dense.

Au surplus, la carte phyto-écologique, renseigne utilement sur les travaux de génie sanitaire à mettre en œuvre et leur « rentabilisation " ultérieure car elle exprime non seulement la salinité des nappes, mais aussi la texture et la microtopographie des milieux. Ainsi, un groupement à Schoenus nigricans indique un sol sableux donc facile à drainer ; à l'inverse, un groupement à Molinia coerula ou Salicornia fruticosa traduisent un sol lourd très imperméable où l'assainissement par drainage sera plus difficile (vocation rizicole ou urbanistique).

Enfin, la carte phyto-écologique se révèle un puissant OUTIL DE RECHERCHE dont on peut attendre, dans l'avenir, les plus brillants résultats.

(8) Les œufs d'Aedes n'éclosent que dans les zones sub-desséchées pendant un certain temps, puis remises en eau. Le repérage permanent des niveaux d'eau suffit à alerter les équipes de prospection au moment des inondations. A l'intérieur d'un ensemble écologique donné, ces fluctuations sont en effet synchrones pour les groupements identiques.

(9) Parmi les facteurs physico-chimiques et biotiques dont peut rendre compte une carte phyto-écologique bien élaborée, citons: la texture du sol, la température relative des eaux, le $p H$ et le degré de pollution. Or, ces facteurs influencent, de manière non négligeable, l'activité des insecticides, en particulier des composés organo-phosphorés. Dans leur étude fondamentale sur l'hydrolyse de ces composés, R. Von Muhlmann et G. Schrader (1957) ont montré que l'hydrolyse augmentait considérablement pour des $p \mathrm{H}$ généralement compris entre 8 et 9 . Les micro-organismes peuvent également jouer un rôle très important dans l'inactivation de ces composés (L. L. Lewallen, 1963, W.-R. Bransby-Williams, 1965). En particulier les insecticides organo-phosphorés appartenant à la série du E 605 (Parathion, Parathion-méthyle, Chlorthion, Isochlorthion, E.P.N., Fenitrothion) pourvus d'un groupement nitré. La réduction de ce groupement par les bactéries (Bacillus subtilis, S. Hirakoso, 1966) ou les organismes fongiques (Hémiascomycètes. E. S. Lichenstein et R. K. Schulz; 1964) entraîne un effondrement de l'activité anti-larvaire :

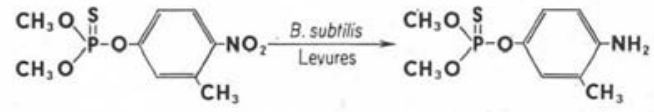

Fenitrothion

Parqe-amino-fenitrothion

Opérant sur Culex pipiens pallens d'une part, Culex pipiens pipiens et Aedes detritus d'autre part, S. Hirakoso, 1966, G. Gras et col., 1966, ont établi que le p-amino-fénitrothion, produit de dégradation, avait une CL 50 supérieure à $100 \mathrm{ppm}$ alors que pour le Fénitrothion cette concentration était habituellement comprise, suivant les auteurs, entre 0,003 et $0,02 \mathrm{ppm}$. 


\section{Bibliographie}

BeCKel (W. E.), 1953. - Preparing mosquito eggs for embryological study. Mosq. New's, 13 (4) : 235-237.

Bransby-Williams (W. R.), 1965. -- Effect of pollution on the susceptibility to larvicides of Culex pipiens fatigans. Bull. O.M.S., 33: 735-737.

Braun-Blanquet (J.), 1953. - Essai sur le classement des biocénoses. Station Intern. Géobot. Méd. Alp. Montpellier. Comm. 118: 8-12.

—, Roussine N. et NÈGRe R., 1951. - Les groupements végétaux de la France Méditerranéenne. Monographie C.N.R.S., 297 p.

Bullock (H. R.), 1960. - Chorionic Pattern of Aedes eggs by sump method. Trans. Am. Micros. Soc., 79 (2) : 16-170.

Clarke (G. L.), 1954. - Elements of ecology. Wiley et Sons, Ed., 534 p.

Clements (A. N.), 1963. - The Physiology of Mosquitoes. Pergamon press Ed., 393 p.

C.N.R.S., 1955. - Les division écologiques du Monde. Moyens d'expression. Nomenclature. Cartographie. Année biologique, $3^{e}$ série, 31 (5-6) : 1-235.

CORRE (J. J.), 1961-1962. - Une zone de terrain salé en bordure de l'Etang de Mauguio. Etude du milieu et de la végétation. Bull. Serv. Carte Phytogéogr., série B, 6 (2) : $105-151$ et 7 (1) : 11-48.

Decoursey (J. D.) et Webster (A. P.), 1952. - A method of clearing the chorion of Aedes sollicitans (Walker) eggs and preliminary observations on their embryonic development. Ann. Ent. Soc. Amer., 45 (4) : 625-632.

Emberger (L.), 1958. - Principes de la méthode de travail du Service de la Carte des Groupements Végétaux du C.N.R.S. Bull. Serv. Carte Phytogéogr., série B, 3 (2): 91-99.

GATEMBY (J. B.) et BeAMS (H. W.), 1950. - The microtomist's vademecum. The Blakiston Company, Ed., 753 p.

Golvan (Y. J.) et Rioux (J. A.), 1961. - Ecologie des Mérions du Kurdistan Iranien. Relations avec l'épidémiologie de la Peste rurale. Ann. Paras. Hum. et Comp., 36 (4): 451-588.

GounOT (M.), 1958. - Contribution à l'étude des groupements végétaux messicoles et rudéraux de la Tunisie. Ann. Serv. Bot. Agr. Tunisie, 31: 152 p.

Graig (G. B.), 1955. - Preparation of the chorion of eggs of Aedine mosquitoes for microscopy. Mosq. News, 15 (4) : 228-231.

- et Horsfall (W. R.), 1958. - Taxonomic and ecological significance of eggs of Aedine mosquitoes. Proc. Tenth. Inter. Congr. Ent. Montreal, 3: 853-857.

Gras (G.), Rioux (J.-A.), Croset (H.), Tour (S.) et Broussal (J.), 1966. - Sensibilité de base d'Aedes (O.) caspius (Pallas) et d'Aedes $(O$.) detritus Halliday à quelques insecticides organo-phosphorés. Bull. Soc. Path. Exot., 1966, 59 (6) : 899-903.

HIRAKoso (S.), 1966. - On the cause of reduction in effectiveness of insecticides in polluted water. Jap. J. Sanit. Zool., 17 (1) : 59-67. 
Horsfall (W. R.), 1956. - A method for making a survey of floodwater mosquitoes. Mosq. News, 16 (2) : 67-71.

- et Craig (G. B.), 1956. - Eggs of floodwater mosquitoes. IV. Species of Aedes common in illinois (Diptera Culicidae). Ann. Ent. Soc. Am., 49 (4) : 368-374.

LEWALLEN (L. L.) et WILDER (W. H.), 1963. - Laboratory tests of insecticides on mosquito larval in polluted and tap water. J. Econ. Ent., 56 (6): 834-835.

Lichenstein (E. P.) et Schulz (K. R.), 1964. - The effect of moisture and micro-organisms on the persistance and metabolism of some organo-phosphorus insecticides in soil with special emphasis on Parathion. J. Econ. Ent., 57 (5) : 618-626.

LoNg (G.), 1958. - Description d'une méthode linéaire pour l'étude de l'évaluation de la végétation. Bull. Serv. Carte Phytogéogr., série B, 3 (2) : 107-126.

LoPP (O. V.), 1957. - Egg sampling as an Index of mosquito breeding. Proc. Forty-four Ann. Meet. New Jersey Mosqu. Exterm. Ass. : 60-63.

MUIRHed-Thomson (R.C.), 1951. - Mosquito behaviour in relation to malaria transmission and control in the tropics. Arnold and Co., Ed., 219 p.

Muhimann Von (R.) et Schrader (G.), 1957. - Hydrolyse der insektiziden Phosphorsäureester Zeitssch. für Nat., 12 (6) : 196-208.

Odum (E. P.), 1959. - Fundamentals of ecology. Saunders, Ed., 546 p.

Popov (G.), ZelleR (W.) et Cocheme (J.), 1965. - Prospection écologique. Rapport sur les études faites en Inde, au Pakistan et en Iran en 1963.64- F.A.O./UNSF/DL/ES/7.

REY (P.), 1957. - L'interprétation des photographies aériennes. Bull. Serv. Carte Phytogéogra. série A, 11 (1) : 5-44.

—, 1964. - Photographies aériennes et végétation. Conférence UNESCO, Toulouse, 1964.

Rioux (J.-A.), 1958. - Les Culicides du « Midi » méditerranéen. Lechevallier, Ed., 303 p.

-, Gras (G.), Croset (H.) et Juminer (B.), 1964. - Essais de lutte anti-culicidienne imagocide par épandage aérien (hélicoptère) de Lindane en aérosols thermiques. Arch. Inst. Pasteur Tunis, 41 (2) : 158-171.

-, Croset (H.), Gras (G.) et Juminer (B.), 1964. - Priorité aux méthodes anti-larvaires dans la lutte contre les Aedes halophiles en Languedoc-Roussillon. (Principes logistiques généraux et application pratique). Arch. Inst. Pasteur Tunis, 41 (3-4) : 386-395.

—, Croset (H.), Gras (G.), Juminer (B.) et Tesson (G.), 1965. - Les problèmes théoriques et pratiques posés par la lutte contre Culex pipiens $\mathrm{L}$. dans le sud de la France. Arch. Inst. Pasteur Tunis, 42 (3-4) : 473-503.

Rossetri (C.), 1964. - Utilisation de la photographie aérienne à grande échelle pour l'étude de la végétation ouverte. Conférence UNESCO, Toulouse, septembre 1964.

SHotwell (R. L.), 1935. - Method for making a grasshopper survey. J. Econ. Ent., 28 : 486-491.

(Travail du Laboratoire d'Ecologie médicale et Pathologie parasitaire

de la Faculté de Médecine; du Laboratoire de Botanique de la Faculté des Sciences;

du Laboratoire de Pharmacie chimique de la Faculté de Pharmacie. E.I.D. 34 -Montpellier) 